\title{
Excited-state band structure mapping
}

\author{
M. Puppin $\odot,{ }^{1,2,{ }^{*}}$ C. W. Nicholson $\odot,{ }^{3}$ C. Monney, ${ }^{3}$ Y. Deng, ${ }^{4}$ R. P. Xian $\odot,{ }^{2}$ J. Feldl $\odot,{ }^{2}$ S. Dong $\odot,{ }^{2}$ A. Dominguez,${ }^{5,6}$ \\ H. Hübener $\odot,{ }^{7}$ A. Rubio, ${ }^{7,8,9}$ M. Wolf, ${ }^{2}$ L. Rettig $\odot,{ }^{2}$ and R. Ernstorfer $\odot^{2,10, \dagger}$ \\ ${ }^{1}$ Laboratoire de Spectroscopie Ultrarapide and Lausanne Centre for Ultrafast Science (LACUS), École Polytechnique Fédérale de Lausanne, \\ ISIC, Station 6, CH-1015 Lausanne, Switzerland \\ ${ }^{2}$ Fritz-Haber-Institut der Max-Planck-Gesellschaft, Faradayweg 4-6, 14195 Berlin, Germany \\ ${ }^{3}$ Department of Physics and Fribourg Center for Nanomaterials, University of Fribourg, Chemin du Musée 3, CH-1700 Switzerland \\ ${ }^{4}$ Paul Scherrer Institute, SwissFEL, 5232 Villigen PSI, Switzerland \\ ${ }^{5}$ Shenzhen JL Computational Science and Applied Research Institute (CSAR), Shenzhen 518110, China \\ ${ }^{6}$ Beijing Computational Research Center (CSRC), Beijing 100193, China \\ ${ }^{7}$ Max Planck Institute for the Structure and Dynamics of Matter and Center for Free Electron Laser Science, Luruper Chaussee 149, \\ Geb. 99 (CFEL), 22761 Hamburg \\ ${ }^{8}$ Center for Computational Quantum Physics, Flatiron Institute, 162 5th Avenue, New York, New York 10010, USA \\ ${ }^{9}$ Nano-Bio Spectroscopy Group, Universidad del Paìs Vasco UPV/EHU, 20018 San Sebastián, Spain \\ ${ }^{10}$ Institut für Optik und Atomare Physik, Technische Universität Berlin, Straße des 17, Juni 135, 10632 Berlin, Germany
}

(Received 17 August 2021; revised 6 December 2021; accepted 26 January 2022; published 17 February 2022)

\begin{abstract}
Angle-resolved photoelectron spectroscopy is an extremely powerful probe of materials to access the occupied electronic structure with energy and momentum resolution. However, it remains blind to those dynamic states above the Fermi level that determine technologically relevant transport properties. In this work we extend band structure mapping into the unoccupied states and across the entire Brillouin zone by using a state-of-the-art high repetition rate, extreme ultraviolet femtosecond light source to probe optically excited samples. The wideranging applicability and power of this approach are demonstrated by measurements on the two-dimensional semiconductor $\mathrm{WSe}_{2}$, where the energy-momentum dispersion of valence and conduction bands are observed in a single experiment. This provides a direct momentum-resolved view, not only on the complete out-of-equilibrium band gap but also on its renormalization induced by electronic screening. Our work establishes a benchmark for measuring the band structure of materials, with direct access to the energy-momentum dispersion of the excited-state spectral function.
\end{abstract}

DOI: 10.1103/PhysRevB.105.075417

\section{INTRODUCTION}

Functionality in electronic and optoelectronic devices is based on the control of the flow of charge carriers under outof-equilibrium conditions. At the microscopic level, charge transport and device operation rely upon generating nonequilibrium electron distributions controlled by external fields to achieve the desired electronic response. The propagation of electrons in a crystal and the evolution of their energy distributions are governed by the details of the electronic structure, as well as the efficiency of elastic and inelastic scattering processes.

Time-resolved angle-resolved photoemission spectroscopy (trARPES) addresses this problem by observing the spec-

\footnotetext{
*michele.puppin@epfl.ch

†ernstorfer@fhi-berlin.mpg.de
}

Published by the American Physical Society under the terms of the Creative Commons Attribution 4.0 International license. Further distribution of this work must maintain attribution to the author(s) and the published article's title, journal citation, and DOI. Open access publication funded by the Max Planck Society. tral function of a material after excitation via a femtosecond optical pulse [1]. The momentum-resolved distribution of excited states combined with the dynamical information on state lifetimes provides a powerful view into excited solids [2], extending the scope of ARPES and allowing to observe out-of-equilibrium electronic properties which can be used to extract the electronic coupling with phonons and other degrees of freedom [3,4]. Ultimately, understanding matter out of equilibrium is mandatory for achieving optical control in complex materials [5]. Time-resolved photoelectron spectroscopy can resolve states unoccupied at equilibrium and has been extensively used to reveal image potential states at surfaces [6,7] and excitons in semiconductors and molecular adsorbates [8,9]. More recently, trARPES was used to reveal the unoccupied band structure of topological materials [10], to measure optically dressed states [11], to observe electron population lifetimes and spin-valley polarization in the conduction band of transition-metal dichalcogenide semiconductors [12-15] and has enabled the direct observation of excitons [2,16,17].

Energy-momentum dispersion of excited states can be determined from trARPES data, both above and below the Fermi level, providing experimental access to quantities such as the 
band-gap [18-20] and conduction-band carrier effect masses [21]. An important open question is how band properties extracted from the trARPES spectral function in the excited state compare with conventional steady-state experiments, e.g., optical spectroscopy or ARPES.

A common expectation is that a comparison is possible in the weak excitation limit [22] where trARPES experiments become very challenging, particularly when accessing the full Brillouin zone (BZ) of the investigated material. This is beyond the reach of most trARPES experiments, which are performed at UV photon energies. Extending these experiments to the XUV photon energy range and correspondingly, to high photoelectron momenta covering the whole BZ, while retaining a comparable signal-to-noise ratio and weak excitation densities, has been challenging until the recent development of suitable high-repetition-rate XUV sources [22-26].

In this work we employ a state-of-the-art experimental setup [23] to simultaneously determine the energy of conduction states (unoccupied at equilibrium) and valence states. This allows us to address the band gap, one of the fundamental optoelectronic properties, by mapping in reciprocal space both valence and conduction bands of $2 \mathrm{H}-\mathrm{WSe}_{2}$, a twodimensional transition-metal dichalcogenide (TMD) semiconductor widely studied for excitonic and spin-valleytronic applications [27-29]. The conduction-band population is probed with a 21.7-eV XUV pulse following photoexcitation by a 3.1-eV pulse, with a temporal resolution better than 100 fs. This enables excited-state ARPES measurements before energy relaxation to the conduction-band minimum, revealing the energy versus momentum dispersion of valence and conduction states in a single experiment, including high-energy conduction electronic states far from the band edge, governing the high-energy optoelectronic properties of TMD semiconductors $[30,31]$. As compared to previous experiments on similar TMD compounds $[12-15,18,20]$, excited-state band mapping is performed in the whole irreducible part of the Brillouin zone, rather than along a finite number of high-symmetry directions, and in a weak excitation regime, where the excited-state band gap and its renormalization due to many-body effects are studied. We demonstrate that in the low-excitation limit the trARPES gap agrees with the band gap measured by other spectroscopies and predicted by theory. This validates excited-state band structure mapping as a generally applicable method to measure, with momentum resolution, the conduction states of materials.

\section{EXCITED-STATE BAND STRUCTURE MAPPING}

To better understand the difference and similarities between ARPES and trARPES, we shortly review the two experimental approaches. In an ARPES experiment, a photon with energy $h v$ excites a single-crystalline sample, and the kinetic energy $E$ of photoelectrons is measured along a wave-vector direction $\mathbf{k}$. If photoionization is treated as a sudden process, the photoemission intensity can be approximated as [32]

$$
I(\mathbf{k}, E)=I_{0}(\mathbf{k}, E) A^{-}(\mathbf{k}, E) f_{\mu, T}(E) .
$$

Equation (1), which for simplicity neglects the experimentally finite angular and energy resolution, as well as charge transport at the surface, links the ARPES spectrum
$I(\mathbf{k}, E)$ to the underlying electronic structure via three factors. The one-electron-removal spectral function, $A^{-}(\mathbf{k}, E)$, contains the information about the quasiparticle band structure and many-body interactions. The spectral weight is modulated by a matrix element term $I_{0}(\mathbf{k}, E)$, which depends on initial- and final-state symmetry and wave vectors, as well as photon energy $(h v)$ and polarization, and the experimental geometry [33,34]. Thirdly, the Fermi-Dirac distribution $f_{\mu, T}(E)$ imposes that only states populated at the temperature $T$ can contribute to the measured spectrum, setting a limit to the highest accessible energy to few $k_{B} T$ above the chemical potential $\mu$. The matrix element term is vanishing unless momentum conservation parallel to the sample's surface is fulfilled by the escaping photoelectron, allowing to link the measured photoelectron angular distribution $I(\mathbf{k}, E)$ to the quasiparticle bands in reciprocal space, as illustrated in Fig. 1(a). Parallel momentum ( $\left.\mathbf{k}_{\|}\right)$conservation, together with energy conservation, imposes that typically only energetic photons in the XUV range can access the whole BZ [35]. As an example, photons with an energy of $\approx 20 \mathrm{eV}$ are necessary to measure the first BZ boundary of $\mathrm{WSe}_{2}$, as indicated by the violet dashed line in Fig. 1(a). In our experiment photoelectron spectra are collected with a hemispherical energy analyzer (HEA) which measures kinetic energy $\left(E_{K}\right)$ and angle of emission along the entrance slit [Fig. 1(b)], which corresponds to a line-cut throughout the function $I(\mathbf{k}, E)$ [full green lines in Fig. 1(a)]. Band mapping is achieved by angular scanning of the sample [green arrows in Figs. 1(a) and 1(b)] across the analyzer slit. The multidimensional function $I(\mathbf{k}, E)$ is constructed from different images, and data can be displayed as constant energy cuts or as energy versus momentum plots, as shown in Fig. 1(a), where a horizontal constant energy cut close to the valence-band maximum and a vertical energy versus momentum dispersion across the BZ are plotted. It is worth noting the alternative approach of momentum microscopy in which the whole accessible photoemission space is collected at the same time [36]. A detailed comparison between the two methods reveals that an HEA ensures higher counting statistics when acquiring data along a specific direction [37], whereas the fixed geometry provided by momentum microscopy is suitable for the study of the symmetry-dependent matrix element $I_{0}(\mathbf{k}, E)$ [34].

A time-resolved ARPES experiment accesses an excited state of the material by performing an ARPES experiment at a well-defined temporal delay $t$ following a femtosecond optical pump pulse [Fig. 1(b)]. The trARPES spectrum $\tilde{I}(\mathbf{k}, E, t)$ thereby measures the (quasi)-electron-removal spectrum as a function of this time delay:

$$
\tilde{I}(\mathbf{k}, E, t)=\tilde{I}_{0}(\mathbf{k}, E, t) \tilde{A}^{-}(\mathbf{k}, E, t) \tilde{f}(\mathbf{k}, E, t) .
$$

Here Eq. (1) is modified to include the explicit time dependence of each term. The optical excitation produces not only an out-of-equilibrium electronic distribution $\tilde{f}$ but also perturbs the many-body interactions in the spectral term $\tilde{A}^{-}$. The matrix element term $\tilde{I}_{0}$ can become a time-dependent quantity if the symmetry of the initial or final states is modified [38]. We follow the convention that for $t>0$ the pump excitation occurs before photoemission: recovery of equilibrium requires that $\tilde{I}(\mathbf{k}, E, t) \stackrel{t \rightarrow+\infty}{\longrightarrow} I(\mathbf{k}, E)$. 

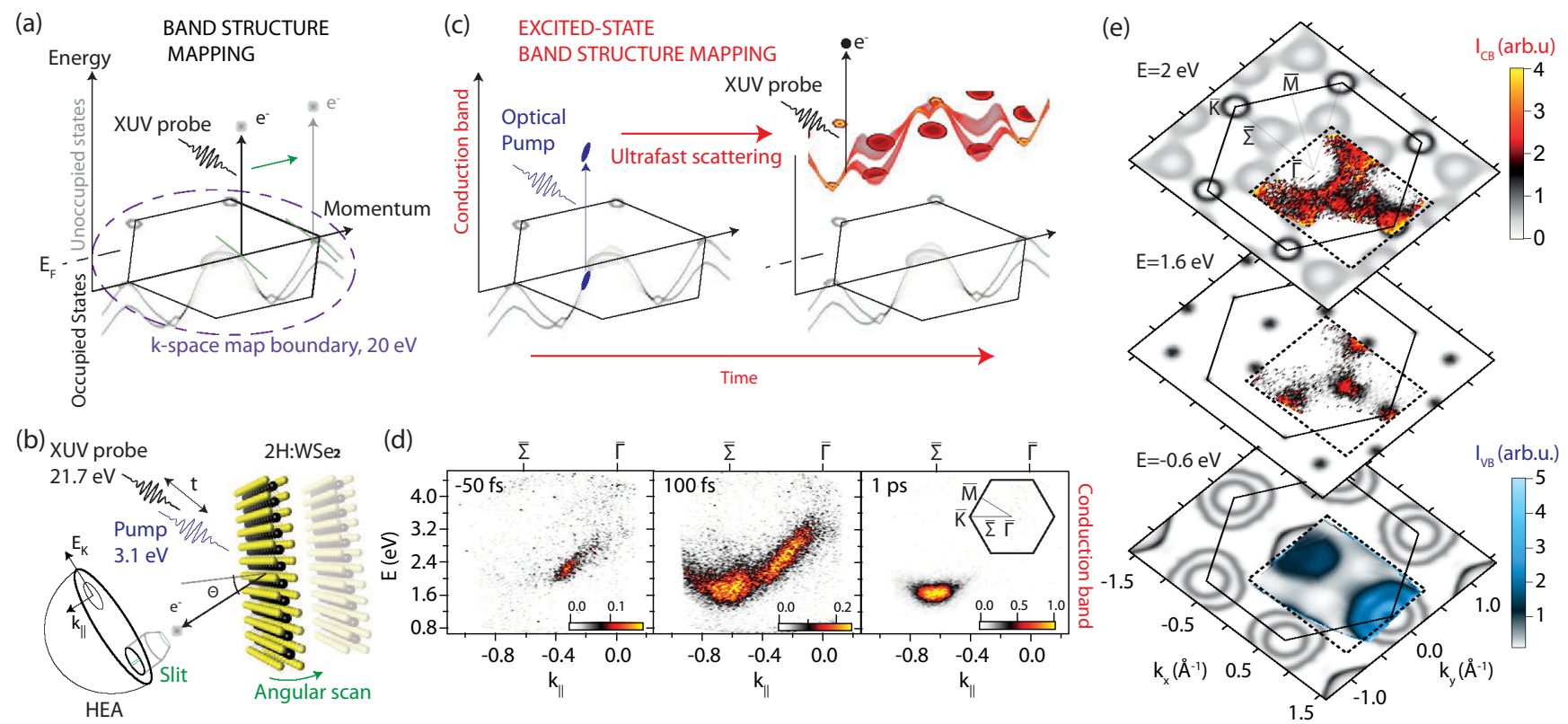

FIG. 1. (a) Band structure mapping in reciprocal space by angle-resolved photoelectron spectroscopy (ARPES). The reciprocal space region measured by the hemispherical energy analyzer (HEA) for two sample tilt angles is indicated by a green line, the maximum parallel momentum which can be accessed by $20 \mathrm{eV}$ photons is indicated by a violet dashed line. (b) trARPES experiments on $2 \mathrm{H}-\mathrm{WSe}{ }_{2}$ : an optical pump pulse at an energy of $3.1 \mathrm{eV}$ excites the system. At a delay $t$, an XUV probe pulse at an energy of $21.7 \mathrm{eV}$ generates photoelectrons, which are measured as a function of the emission angle $\theta$ with a HEA. The sample angle is scanned across the analyzer slit to collect ARPES maps. (c) Excited-state band structure mapping. (d) trARPES data collected in the conduction band of WSe ${ }_{2}$ for pump-probe delays of -50 fs, $100 \mathrm{fs}$, and 1 ps. Inset: The surface Brillouin zone of $\mathrm{WSe}_{2}$. (e) Photoelectron intensity distribution as a function of parallel momentum for three energies at a pump-probe delay of $100 \mathrm{fs}$; VB and CB energy distribution curves have been independently intensity normalized for better visualization. The experimental data is collected in a region delimited by the dashed line. Outside this region, the results of $\mathrm{G}_{0} \mathrm{~W}_{0} \mathrm{calculations}$ are displayed, and the theoretical band dispersion along the $k_{z}$ direction was integrated; the conduction bands were rigidly offset by a scissor operator of $-0.16 \mathrm{eV}$ to match the experimental energy.

As illustrated in Fig. 1(b), trARPES provides access to states unoccupied at equilibrium. This can be understood as a two-step process, where in a first step the femtosecond pump pulse creates an optical polarization in allowed momentum and energy regions corresponding to vertical optical transitions in the material $(\Delta \mathbf{k}=0)$ [39]. In a second step, microscopic scattering events within a few hundred femtoseconds redistribute the electronic population to multiple states across the conduction band (CB) [Fig. 1(a)]. Electrons relax their excess energy via multiple electron-phonon scattering events towards the band edges and accumulate at the $\mathrm{CB}$ minima on timescales typically shorter than a few picoseconds. By measuring the photoelectron energy and angular distribution before significant energy relaxation to the lattice has occurred, the information encoded in $\tilde{A}^{-}$can be revealed in a range $E<\mu+h v_{p}$, where $h v_{p}$ is the pump photon energy.

Excited-state band mapping of unoccupied states is particularly demanding and strongly benefits from high-repetitionrate $(>100 \mathrm{kHz})$ XUV sources. First, a sufficiently short XUV pulse is fundamental for accessing the out-of-equilibrium state before its decay throughout the BZ. In addition, space charge effects, which are inherent in ARPES with short XUV pulses, are mitigated in high-repetition-rate experiments [40]. Furthermore, the higher the pump excitation energy density, the stronger many-body interactions modify the function $\tilde{I}(\mathbf{k}, E, t)$ relative to the equilibrium case. trARPES experiments at high repetition rates benefit from higher counting statistics and hence data can be acquired at weaker perturbation strengths.

\section{EXPERIMENTAL METHODS}

To meet the simultaneous requirements of an ultrashort XUV source with a high repetition rate, in this work we generate probe pulses by high-harmonic generation with an optical parametric chirped pulse amplifier operating at $500 \mathrm{kHz}$ [41]. This results in XUV pulses at an energy of $21.7 \mathrm{eV}$ and with characteristic time-bandwidth products of approximately $20 \mathrm{fs} \times 110 \mathrm{meV}$ [23], which are temporally short enough to access the excited states before significant carrier energy relaxation has occurred and, at the same time, have an energy bandwidth sufficiently narrow to resolve the excited-state energy features. trARPES experiments were performed on single-crystalline samples of bulk $\mathrm{WSe}_{2}$ cleaved in ultrahigh vacuum conditions. Commercial $\mathrm{WSe}_{2}$ single crystals where prepared by exfoliation in situ under UHV conditions. The base pressure during the experiments was below $1 \times 10^{-10}$ mbar. The material was excited by a pump pulse with a photon energy of $3.1 \mathrm{eV}$ and at an excitation energy density of $40 \mu \mathrm{J} / \mathrm{cm}^{2}$. All the experiments were performed at room temperature, where no surface photovoltage or charging effects were observed.

To illustrate the ability of trARPES to visualize states which are unoccupied at equilibrium, we show in Fig. 1(d) 
energy versus momentum data collected in an energy window in the CB along the high-symmetry direction $\bar{\Gamma}-\bar{K}$. Three selected time delays ( $-50 \mathrm{fs}, 100 \mathrm{fs}$, and $1 \mathrm{ps}$ ) are plotted side by side. The surface BZ of $\mathrm{WSe}_{2}$, with the high-symmetry points marked, is shown as an inset of Fig. 1(d). During the rising edge of the pump pulse ( $-50 \mathrm{fs}$ ), the $\mathrm{CB}$ signal is localized at $-0.35 \AA^{-1}$ from the BZ center ( $\bar{\Gamma}$ point $)$. This suggests that in this region population is transferred via an optical transition at the photon energy of $3.1 \mathrm{eV}$ rather than indirectly by scattering. The intensity of this feature as a function of time was used as a measure of the pump-probe temporal cross-correlation, and the temporal maximum was used to define the time zero. The FWHM of the cross-correlation is $95 \mathrm{fs}$, dominated by the pump pulse duration. Further details concerning the crosscorrelation fits are shown in the Appendix. Throughout this work, the zero energy was set for convenience to the valenceband energy at the $\bar{K}$ point, the corner of the hexagonal BZ.

At a time delay of $100 \mathrm{fs}$, population can be observed throughout the conduction states, up to at an energy $\approx 2.5 \mathrm{eV}$ [Fig. 1(d), central panel]. This delay was selected to perform the excited-state band structure mapping. Relaxation towards the $\Sigma$ conduction-band valley minimum is indeed already apparent at a delay of 1 ps [Fig. 1(d), right panel].

An energy window from -1.5 to $3.5 \mathrm{eV}$ was selected to simultaneously observe valence and conduction bands around the band gap, which is a unique feature of trARPES. Three exemplary constant energy cuts of the data at $t=100 \mathrm{fs}$ are shown in Fig. 1(e), which display in false colors the photoelectron intensity distribution as a function of parallel momentum for energies of $-0.6 \mathrm{eV}$ in the valence band (VB), $1.6 \mathrm{eV}$ and $2 \mathrm{eV}$ in the conduction band (CB). The measurement region is indicated by a dashed line and comprises the whole first BZ of $\mathrm{WSe}_{2}$. In Fig. 1(e), two different false color scales are used for conduction and valence states. Energy distribution curves (EDCs) in the VB were normalized to the same area as a function of parallel momentum. This was chosen for reducing the impact of the matrix element in the display of the constant energy map and for a clearer comparison with theoretical calculations. The same procedure was applied independently to EDCs in the $\mathrm{CB}$ (i.e., on the data for $\mathrm{E}>1 \mathrm{eV}$ ), but prior to the area normalization, an exponential background tail from the underlying occupied states was subtracted. No normalization procedure was performed on the data displayed in the other figures of the text.

\section{RESULTS AND DISCUSSION}

To rationalize the experimental data we perform $a b$ initio density functional theory (DFT) calculations of the electronic band structure. The system was modeled using a hexagonal supercell with the experimental lattice constants $a=b=3.28 \AA$ and $c=12.98 \AA$ [42]. DFT calculations were performed using the generalized gradient approximation (GGA) with the Perdew-Burke-Ernzerhof (PBE) functional [43], as implemented in the QUANTUM ESPRESSO package [44]. To improve the agreement with experimental data, we use many-body perturbation theory at the one-shot $G_{0} W_{0}$ level $[45,46]$ on top of the DFT results. The Brillouin zone was sampled with a $9 \times 9 \times 9 k$-point grid, and the spin-orbit coupling was included directly in the DFT calculations and perturbatively at the $\mathrm{G}_{0} \mathrm{~W}_{0}$ level using the BerkeleyGW package [47]. Finally, we performed DFT calculations using a $24 \times 24 \times 9$ BZ sampling and interpolated linearly the $9 \times 9 \times 9$ GW band structure into this finer $k$-point grid. We used a total of 1000 conduction bands and a 18 Ry energy cutoff for the computation of the inverse dielectric matrix. For the evaluation of the screened and bare Coulomb parts of the self-energy operator, we used energy cutoffs of 18 Ry and 160 Ry, respectively. All employed cutoff values, BZ sampling, and number of bands were systematically and independently increased until results were converged within a few tens of $\mathrm{meV}$ for the conduction- and valence-band energy difference. The $\mathrm{G}_{0} \mathrm{~W}_{0}$ method computes quasiparticle energies, correcting to lowest order the unscreened electronic Green's function $G_{0}$ by the Coulomb interaction $W_{0}$. The quasiparticle energy dispersion is calculated as a function of the three-dimensional wave vector $\left(k_{x}, k_{y}, k_{z}\right)$. For a direct comparison with data in Fig. 1(d), the theoretical bands are integrated along the reciprocal space direction orthogonal to the sample surface $\left(k_{z}\right)$. This choice is justified by the strong surface sensitivity of XUV-based photoemission due to the short mean free path of photoelectrons. Electron momentum conservation is relaxed for the $k_{z}$ component, adding an additional source of energy broadening for bands with dispersion out of the surface plane. There is strong evidence that in $\mathrm{WSe}_{2}$ the photoemission probing depth at $21.7 \mathrm{eV}$ is mostly limited to the uppermost layer $(\approx 0.5 \mathrm{~nm})$, in fact, inversion-symmetric $\mathrm{WSe}_{2}$ surprisingly exhibits strong spin-polarized bands [48] and valley polarization in circularly pumped tr-ARPES [15]. The importance of final-state effects in the material is evidenced by one-step photoemission calculations [34] and will be discussed further below.

The experimental data contains the excited-state $\mathrm{CB}$ and VB energy-momentum dispersion for arbitrary reciprocal space directions, which can be compared with our $a b$ initio calculations and with other experiments. For this purpose, energy versus momentum photoelectron distributions are plotted along three high-symmetry directions $\bar{\Gamma}-\bar{\Sigma}-\bar{K}, \bar{K}-\bar{M}, \bar{M}-\bar{\Gamma}$ in Fig. 2 and compared with the results of the calculations. The theoretical $k_{z}$ dispersion is indicated by a shading, highlighting two-dimensional (low- $k_{z}$ dispersion) and threedimensional states. The experimental photoelectron intensity is plotted without additional normalization, and intensity modulations are attributable to the momentum-dependent matrix element. The average intensity of the conduction-band signal is a factor $10^{-3}$ that of the valence states, and we use two distinct false color scales for conduction and valence states, respectively.

The zero energy reference is set to the highest energy VB at the $\bar{K}$ point also for the theoretical data to minimize any alignment uncertainty due to $k_{z}$ dispersion. The theoretical conduction states were shifted by $-160 \mathrm{meV}$ to match the measured CB energy at the $\bar{K}$ point, both in Fig. 1(d) and in Fig. 2; the same energy shift was applied for every electron momentum (rigid scissor operator).

Theory predicts two valence and two conduction bands in the observed energy window, as all calculated bands are spin degenerate, consistent with the inversion-symmetric bulk crystal structure of $2 \mathrm{H}-\mathrm{WSe}_{2}$. The spin-orbit splitting of the VB band at the $\bar{K}$ point is $\approx 500 \mathrm{meV}$, in good agreement 


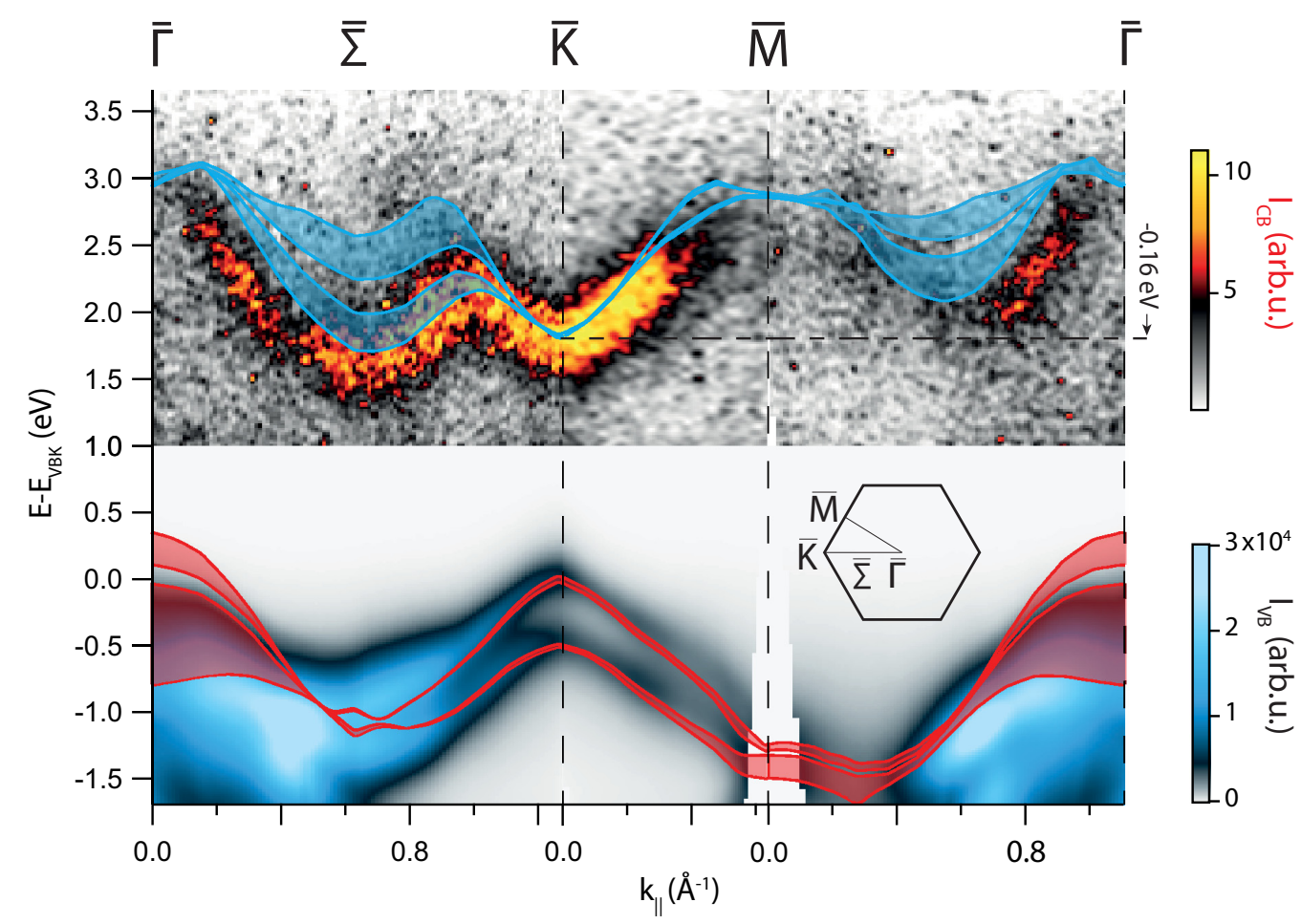

FIG. 2. Measured ARPES intensity as a function of energy and parallel momentum showing the VB and CB along the $\bar{\Gamma}-\bar{\Sigma}-\bar{K}, \bar{K}-\bar{M}$, $\bar{M}-\bar{\Gamma}$ directions, indicated in the upper panel. Conduction-band states are displayed by a different color scale. Blue and red curves indicate the quasiparticle energies calculated with the $\mathrm{G}_{0} \mathrm{~W}_{0}$ method for the $\mathrm{CB}$ and $\mathrm{VB}$, respectively. The theoretical band structure energy zero was set to the VB position at the $\bar{K}$ point, and the CBs (blue) were rigidly shifted by a $-0.16-\mathrm{eV}$ scissor operator to match the $\bar{\Sigma}$ valley center energy. The momentum dispersion along the $k_{z}$ direction is indicated by the shaded area.

with past literature [49-51]. Despite being a layered quasi-2D material, $\mathrm{WSe}_{2}$ displays some inherently three-dimensional features. In particular, the $\Sigma$ valley, as well as the valence band at the $\bar{\Gamma}$ point, have considerable $k_{z}$ dispersion. In contrast, the out-of-plane band dispersion is low in the vicinity of the $\bar{K}$ point, as confirmed by energetically narrower features in ARPES. Our $\mathrm{G}_{0} \mathrm{~W}_{0}$ calculations predict an orthogonal momentum dispersion on the order of $40 \mathrm{meV}$ for the $\mathrm{VB}$ and $30 \mathrm{meV}$ for the $\mathrm{CB}$ at the $\bar{K}$ point. Calculations place the indirect band gap between the maximum of the VB at the $\bar{\Gamma}$ point and the $\Sigma$ valley. In our data the conduction-band minimum $(\mathrm{CBM})$ is unambiguously located at the $\bar{\Sigma}$ point; however, the apparent valence-band maximum (VBM) is observed at the $\bar{K}$ point, and a broad continuum of states is observed at the $\bar{\Gamma}$ point. It is widely accepted that the absolute VB maximum is located at the $\bar{\Gamma}$ point and that matrix element effects cancel the contribution of the upper VB at $\bar{\Gamma}[48,49]$. After the rigid offset of $-160 \mathrm{meV}$ mentioned above, the $\mathrm{G}_{0} \mathrm{~W}_{0}$ calculations are in qualitative agreement with the excited-state band structure and reproduce the main features of the experimental conduction band.

For a quantitative comparison, the quasiparticle energy must be determined from the ARPES intensity. Final-state effects usually complicate the retrieval of quasiparticle energies and of many-body effects in the spectral function. However, the problem is absent in a strictly two-dimensional state [dispersion only along $\mathbf{k}_{\|}=\left(k_{x}, k_{y}\right)$ ] [52]. Both valence and conduction states at the direct optical band gap at the $\bar{K}$ point are quasi-two-dimensional, enabling a robust comparison of the experimental excited-state band gap with theory and other experimental techniques.

The CB and VB energies are extracted from the experimental data by a fit of the energy distribution curve at the $\bar{K}$ pointfor $t=100 \mathrm{fs}$. The procedure is illustrated in Fig. 3(a); the photoelectron spectrum of the VB is well fitted by two Gaussian peaks and by a Shirley background. The two, nearly degenerate conduction bands predicted by theory are not resolved within the experimental linewidth, and a single Gaussian peak describes well the CB signal. Due to its higher intensity, the higher energy tail of the VB spectrum appears as a background on the $\mathrm{CB}$ and is modeled by an exponential decay. We define the experimental band gap as the distance between the uppermost VB peak position $(E=0$ by definition) to the center of the CB peak, as highlighted by the red line in Fig. 3(a), and we measure a band gap of $1.78 \pm 0.03 \mathrm{eV}$ for data collected at a fluence of $40 \mu \mathrm{J} / \mathrm{cm}^{2}$. We note that this procedure, valid for quasi-2D bands, differs from the method adopted for three-dimensional semiconductors, where the band edge is found by linear extrapolation of the photoelectron spectral edge [53]. The excited-state quasiparticle energy, an out-of-equilibrium quantity, can change as a function of the excitation energy density [18-20]. To investigate the impact on the band gap, we follow its evolution for increasing incident optical energy density up to $320 \mu \mathrm{J} / \mathrm{cm}^{2}$ and observe a decrease of the band gap [Fig. 3(b)]. The maximum effect is $\approx 50 \mathrm{meV}$, with a linear slope of $-2 \pm 1 \times 10^{-1} \mathrm{meV} /\left(\mu \mathrm{J} / \mathrm{cm}^{2}\right)$; the extrapolated limit at zero excitation density is $1.77 \pm 0.01 \mathrm{eV}$. 
(a)

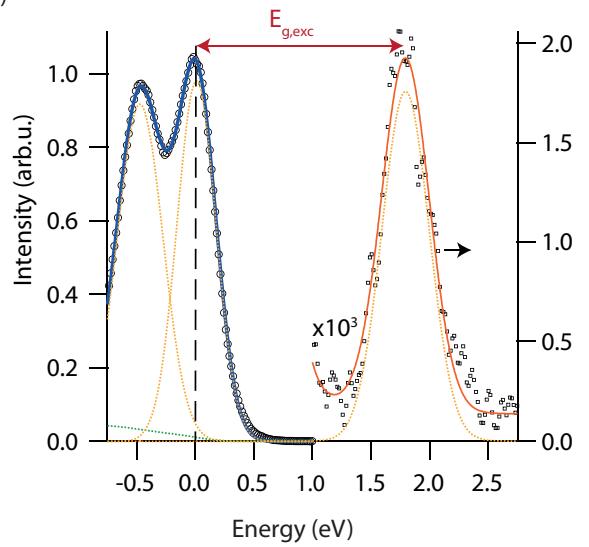

(b)

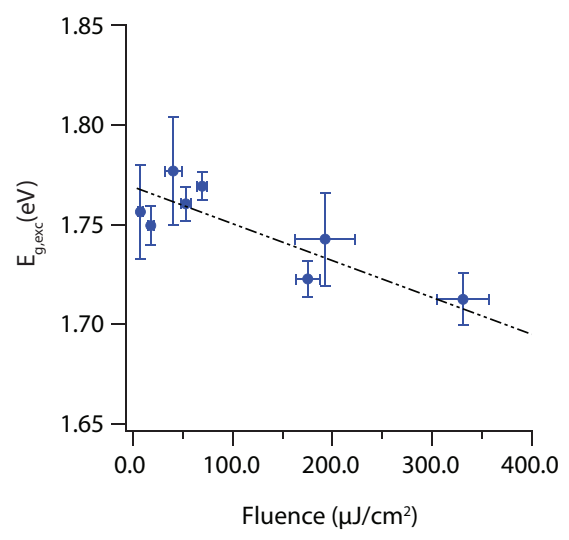

(c) FUNDAMENTAL GAP

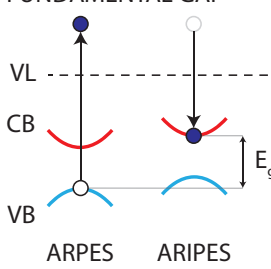

(e) EXCITED-STATE GAP

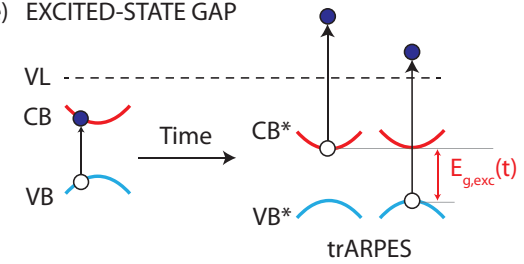

FIG. 3. (a) Energy distribution curve at the $K$ point, together with the fit used to determine the excited-state band gap $E_{g \text {,exc }}$. The conductionband signal intensity, displayed on the right-hand axis, was scaled by a factor $10^{3}$ for clarity. (b) Fluence dependence of the excited-state direct band gap at the $\bar{K}$ pointfor a time delay of $100 \mathrm{fs}$. Schematic description of the (c) fundamental, (d) optical, and (e) excited-state direct band gaps at the $\bar{K}$ valley, where VL indicates the vacuum level. Only the direct gap is considered, i.e., the energy of emitted (absorbed) electrons is measured at the same parallel momentum value. The photoexcitation occurs at time zero at a different reciprocal space location, above the direct band gap. The photoexcited electron and hole distributions renormalize the excited-state bands, indicated by $\mathrm{CB}^{*}$ and $\mathrm{VB}^{*}$.

It is interesting to compare this experimental band gap, which we call the excited-state band gap $E_{g, \text { exc }}$, with $a b$ initio calculations and other experimental techniques. Several experiments have been designed to resolve the electronic structure above the chemical potential [54]. Inverse photoemission [55], scanning tunneling spectroscopy [56], and very low-energy electron diffraction [57] access unoccupied conduction states by adding an electron to the system and probing the complementary one-electron-addition spectral function $A^{+}(\mathbf{k}, E)$ [58]. Angle-resolved inverse photoemission (ARIPES), in particular, has momentum resolution [54]. Unfortunately, due to the small cross-section of the process and, unlike ARPES, due to the lack of parallel detectors with multiple angular and energy channels, ARIPES has not evolved to a similarly widespread technique [55]. Another approach can used in photoemission to observe otherwise unoccupied states, namely, sample doping by alkali metal atoms [50,59]. A limitation of alkali doping is the possibility of chemical modification to the band structure [50]. Additionally, resonant inelastic x-ray scattering techniques have also been used to map the dispersion of unoccupied states $[60,61]$. The direct gap at the $\bar{K}$ point for $\mathrm{WSe}_{2}$ from various methods is displayed in Table I.

The fundamental or quasiparticle band gap $E_{g, f}$ is usually defined as the difference between the electron affinity, i.e., the energy gained by adding a single electron to an $\mathrm{N}$ electron system, and the ionization energy, needed to remove an electron leaving $\mathrm{N}-1$ electrons behind [74]. The quasiparticle gap should not be confused with the so-called optical band gap, which will be discussed later on. The socalled transport band gap, determined by electrical transport measurements, coincides with the fundamental band gap; however, in the case of semiconductors such as bulk $\mathrm{WSe}_{2}$, possessing an indirect band gap and multiple conductionband valleys, momentum-resolved techniques provide a more complete picture. In view of comparison with optical spectroscopy, we restrict discussion here to the case of the direct band gap at the $\bar{K}$ valley and more loosely consider the band gap as a momentum-dependent quantity which attains its minimum at the direct fundamental band gap. Experimentally, the momentum-dependent quasiparticle band gap can be measured by comparing the VB measured by photoemission ( $N-1$ electron final state) with the CB measured by inverse photoemission $(N+1$ electron final state). This procedure is schematized in Fig. 3(c) and necessitates a common energy reference between the two experimental setups. In particular, the direct fundamental gap of $\mathrm{WSe}_{2}$ at the $\bar{K}$ point was experimentally measured to be $E_{g, f}^{\exp }=1.7 \pm 0.1 \mathrm{eV}$ by combining ARPES and ARIPES [49].

When comparing the experimental gap with theoretical results, an important question is to what extent one is allowed to compare $a b$ initio calculations such as DFT with energies determined by (time-resolved) photoelectron spectroscopy. DFT computes the ground-state electronic density and returns a set of self-consistent Kohn-Sham (KS) bands [75]. Even in an idealized case where the exact density functional is known, a direct comparison between the KS bands and the

TABLE I. Comparison between experimental (upper part) and theoretical direct band gap of $\mathrm{WSe}_{2}$ (lower part) at the $\bar{K}$ point. ${ }^{*}$ Measured at $77 \mathrm{~K}$; at room temperature the gap is reduced by $\approx 60 \mathrm{meV}$ [65]. ${ }^{\star}$ bilayer $\mathrm{WSe}_{2}$.

\begin{tabular}{lcc}
\hline \hline Method & Band gap $(\mathrm{eV})$ & References \\
\hline ARPES+ARIPES & $1.7,1.4$ & {$[49],[62]$} \\
ARPES+Doping & 1.62 & {$[59]$} \\
trARPES & 1.77 & This work \\
Optics, A exciton & $1.697^{*}, 1.60,1.626$ & {$[63],[64],[65]$} \\
Optics, Interband & $1.752^{*}, 1.686$ & {$[63],[65]$} \\
EELS, A exciton & 1.75 & {$[66]$} \\
DFT & $1.25,1.17-1.55$ & This work, [67-71] \\
$G_{0} W_{0}$ & $1.90,1.75,2.08^{\ddagger}$ & This work, [72], [73] \\
BSE, A exciton & $1.86^{\ddagger}$ & {$[73]$} \\
BSE, Interband & $2.02^{\ddagger}$ & {$[73]$} \\
\hline \hline
\end{tabular}


ARPES measurements is not justified [76]. Nonetheless, in many cases, within a constant energy offset, the KS bands are in good agreement with ARPES data of the valence band. For $\mathrm{WSe}_{2}$, in particular, DFT bands reproduce reasonably well the ARPES VB energy dispersion [48,49,51,77]. However, if $E_{g, f}$ is directly calculated from the KS bands, theory grossly underestimates the band gap. Before applying the $\mathrm{G}_{0} \mathrm{~W}_{0}$ correction, our calculations predict a gap value of $1.25 \mathrm{eV}$, in line with other DFT results, reported in Table I. This wellknown band-gap problem is intrinsic to DFT [78] and is a reminder that $\mathrm{KS}$ energies are indeed not quasiparticle energies. Conversely, Hedin's GW method [46,79] can be used to calculate quasiparticle excitations in a solid, such as measured in ARPES (electron removal) or ARIPES (electron addition). GW calculations correct the DFT energies by an approximate electronic self-energy, typically performed to the lowest order $\left(\mathrm{G}_{0} \mathrm{~W}_{0}\right)$. We find a considerable improvement in the calculated fundamental gap and obtain a value $E_{g, f}^{G W}=1.90 \mathrm{eV}$, in line with previous calculations [72].

A second commonly defined band gap is the so-called optical band gap $E_{g, o}$, which corresponds to the lowest energy required for a vertical $(\Delta \mathbf{k}=0)$ electronic transition in the system. This is a neutral excitation where both the initial and final states have $N$ electrons, in contrast with the case of the fundamental gap, which is calculated as the energy difference between an $N+1$ and an $N-1$ electron state. The optical band gap can be experimentally measured by optical absorption spectroscopy. A remarkable feature in optical absorption spectra is the appearance of excitonic resonances at energies below the onset of electronic interband transitions, as shown in Fig. 3(c). The observation of an excitonic peak is the hallmark of the electron-hole interaction. To predict the optical absorption spectrum $a b$ initio, one must solve the Bethe-Salpeter equation [80]. In the optical absorption spectra of bulk $\mathrm{WSe}_{2}$ the so-called A exciton is the lowest resonance at an energy of $1.68 \mathrm{eV}$, the exciton binding energy $E_{x}$ was determined to be $50 \mathrm{meV}$, and the interband transition has an energy of $1.73 \mathrm{eV}$ [63]. This sets the scale for the electron-hole interaction in bulk TMD semiconductors, and one expects $E_{g, o} \approx E_{g, f}-E_{x}$.

In the excited-state band-gap measurement [Fig. 3(d)], a neutral optical excitation is followed by an ionization step at time $t$, leading to a $N-1$ electron excited final state with an additional hole in the $\mathrm{VB}$, which is generated for $t=0$ and is followed by a relaxation dynamics for $t>0$. The band gap is measured by comparing the kinetic energy of photoelectrons originating from the $\mathrm{CB}$ and the VB. Generally speaking, $E_{g \text {,exc }}(t)$ is a time-dependent quantity influenced by manybody effects and can be renormalized by electron-electron interactions, leading to screening and excitonic effects, and by the electron-phonon coupling with the (nonthermal) phonon distribution.

Our data shows that in the low-excitation limit, $E_{g, \text { exc }}(100$ fs) is in good numerical agreement with the fundamental band gap determined by other experiments. Furthermore, we observe no signatures of the A excitonic peak at the $\bar{K}$ point, which appears in optical measurements at a lower energy of $\approx 1.62 \mathrm{eV}$ [63-65]. A deviation from the single-quasiparticle picture is expected when electron and hole are bound to form excitons [81-83] and photoelectron spectra bear the signature of such interactions as a renormalized energy and momentum dispersion [2,7]. The agreement with the theoretical $\mathrm{G}_{0} \mathrm{~W}_{0}$ bands in the present case can be rationalized by the fact that the pump photon energy is well above the gap and sufficiently off-resonance to approximate the initial $(t \approx 0)$ carrier distribution as an electron-hole plasma, where exciton quasiparticles are not formed [39]. In bulk $\mathrm{WSe}_{2}$ the formation of stable A excitons at the $\bar{K}$ point is hindered by the possibility of electron (hole) scattering to the $\bar{\Sigma}$ point ( $\bar{\Gamma}$ point), which are the global band energy edges. However, if instead the excitation energy is resonant with the excitonic peak observed by optics, excitonic effects can be observed [2].

We note that $\mathrm{G}_{0} \mathrm{~W}_{0}$ calculations overestimate the band gap observed in our out-of-equilibrium experiment by $\approx 160 \mathrm{meV}$. However, the agreement with the observed band dispersion is still satisfactory upon a rigid shift of the conduction bands to lower photon energies, suggesting that a single-quasiparticle picture holds well for the excited-state band structure in first approximation. Band-gap renormalization is expected to occur due to carrier screening and via electron-phonon coupling $[18,19,84]$. Time-resolved diffraction studies reveal that a nonequilibrium phonon distribution rises on the timescale of a few picoseconds [85]. At a pump-probe delay of $100 \mathrm{fs}$, where our data was collected, a significant hot phonon population has not yet developed. We conclude that electronic screening must dominate in band structure mapping experiments, and we attribute to this effect the observed band-gap reduction at higher excitation densities [Fig. 3(b)].

Having established that the excited-state band gap well approximates the fundamental band gap in our experimental conditions, we now extract the momentum-resolved energy dispersion contained in the experimental maps for the whole 2D $\bar{K}$ valley. The $\bar{K}$ valley energy is shown in Fig. 4(a), and for comparison we plot the theoretical dispersion of the lowest CB in Fig. 4(b). The threefold symmetry of the valley is evident from the data, and the anisotropy of the $\bar{K}$ valley can be quantified by extracting the dispersion along the highsymmetry directions K- $\Sigma$ and K-M, indicated in Fig. 4(b). For this purpose we employ the previously described fitting procedure to EDCs surrounding the $\bar{K}$ valley. The band dispersion of both conduction and valence bands was estimated by fitting a parabola in a range of $0.15 \AA^{-1}$, as illustrated in Fig. 4(c) for the case of the CB. We obtain a value of $m_{e}^{K \Sigma}=0.38 m_{0}$ $\left(m_{h}^{K \Sigma}=-0.52 m_{0}\right)$ and $m_{e}^{K M}=0.55 m_{0}\left(m_{h}^{K M}=-0.56 m_{0}\right)$ for the $\mathrm{CB}(\mathrm{VB})$ in the directions $\mathrm{K}-\Sigma$ and $\mathrm{K}-\mathrm{M}$, respectively, where $m_{0}$ is the electron mass. The experimental dispersion is somewhat smaller than effective masses reported for DFT, $m_{h}=-0.625 m_{0}$ and $m_{e}=0.821 m_{0}$ [86]. Calculated effective masses from DFT depend strongly on computational details and also on the computational band gap [87]; larger theoretical masses might be therefore linked to the underestimation of the gap in the aforementioned work.

By observing the hole and electron quasiparticle independently, one can calculate effective $\left[M=m_{e}+m_{h}\right]$ and reduced $\left[\mu_{r}=m_{e} m_{h} /\left(m_{e}+m_{h}\right)\right]$ exciton masses. The exciton effective masses are $M^{K \Sigma}=0.9 m_{0}$ and $M^{K M}=1.1 m_{0}$, which can be compared with experimental results from electron energy loss spectroscopy, $M=0.91 m_{0}$ [66], and with optical measurements under a magnetic field, which report $M=0.7 m_{0}$ [88]. The exciton reduced mass determined 

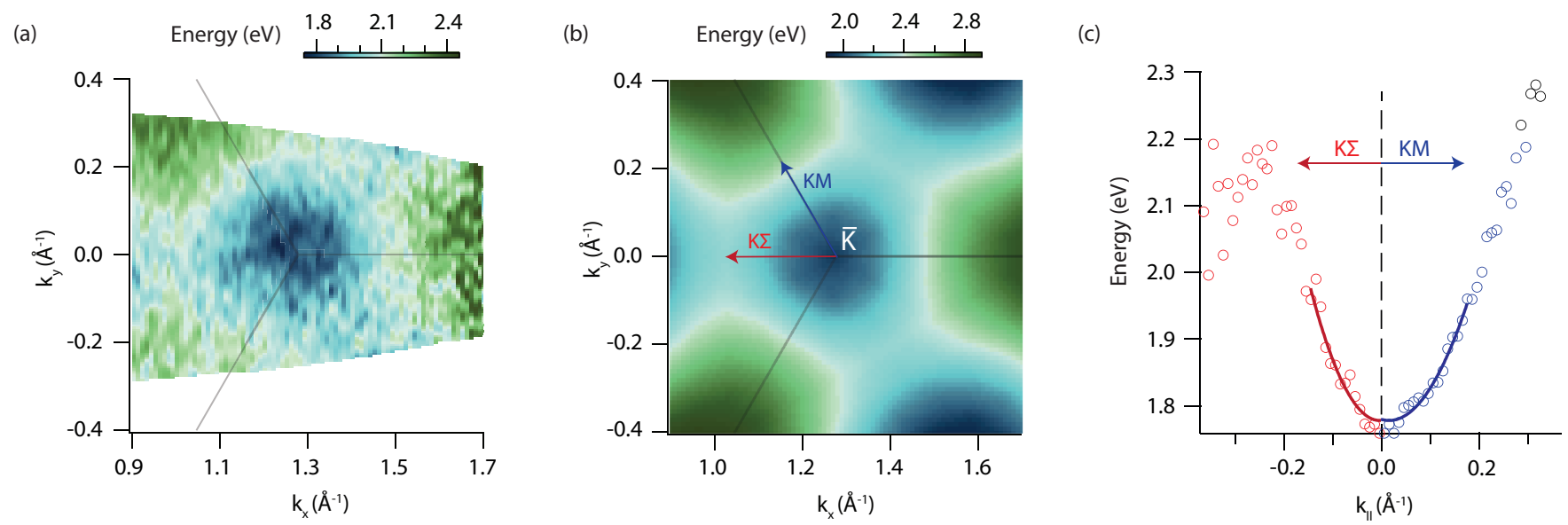

FIG. 4. (a) Conduction-band center energy at the $\bar{K}$ valley, (b) $\mathrm{G}_{0} \mathrm{~W}_{0}$ energy of the $\mathrm{K}$ valley, and (c) dispersion along the directions $\mathrm{K}-\Sigma$ (negative $x$ axis) and K-M (positive $x$ axis). The full line indicates the result of parabolic fits to the data.

from our data is $\mu_{r}^{K \Sigma}=0.22 m_{0}$ and $\mu_{r}^{K M}=0.28 m_{0}$. This can be compared with optical absorption spectroscopy data from which $\mu_{r}=0.21 m_{0}$ was determined [63]. We stress, however, that despite the reasonable numerical agreement, other techniques do not identify the hole and electron masses independently. Furthermore, band anisotropy along different symmetry directions can be readily identified and accounted for within the excited-state band structure. This is particularly relevant, for example, in valleytronic applications in heterolayers, where energy-degenerate valleys appear at different momentum locations [89]. The detailed effects of layer stacking on the momentum dispersion and on the optical and transport properties is as yet poorly understood and can be directly characterized by excited-state band structure mapping.

\section{CONCLUSIONS}

The excited-state band structure is visualized for the TMD $\mathrm{WSe}_{2}$ by tr-ARPES. The experiment provides simultaneous access to valence and conduction states throughout the BZ, thereby completely mapping the material's band gap. The excited-state direct gap at the $\bar{K}$ point agrees in the low-excitation limit with fundamental quasiparticle gap, as obtained by static experiments. Our experiment shows that the excited-state band structure agrees in the low-excitation limit with the single-quasiparticle bands, and we obtain experimentally conduction- and valence-band dispersion for the $\bar{K}$ point for various high-symmetry directions. Thanks to XUV light sources at high repetition rate, we anticipate that the measurement of the excited-state band structure in the whole BZ can be performed for a broad class of samples. $\mathrm{G}_{0} \mathrm{~W}_{0}$ calculations provide a good qualitative description of the data but predict the experimental out-of-equilibrium band gap only within $160 \mathrm{meV}$. Excite-state band structure mapping can provide an experimental benchmark to quantitatively fine tune computations, e.g., to accurately predict the band gap in high-throughput computational material discovery for optoelectronic applications [71,90]. Automated methods for comparison with theory, demonstrated for multidimensional ARPES data [91], are applicable also to excited-state band structure data. Importantly, the method provide access to un- occupied states of quantum materials to resolve topological features above the Fermi level [10], and for charge density wave materials, where the excited-state band structure can be followed across a photon-induced phase transition [92-94]. A future open question is the applicability of the method for strongly correlated materials, e.g., to access the spectral function of unoccupied states in correlated oxides, to reveal the symmetry of the momentum distribution of the upper Hubbard bands in cuprate superconductors [95]. Calculations out of equilibrium in such systems is a challenge for current theoretical methods [96], and a detailed knowledge of the unoccupied states is lacking. In this case, short-lived excitedstate features might be accessible across the Brillouin zone by suitably tuning the time-bandwidth product to improve the temporal resolution, which is necessary to fully exploit excite-state band structure mapping.

\section{ACKNOWLEDGMENTS}

This work was funded by the Max-Planck-Gesellschaft, by the German Research Foundation (DFG), within the Emmy Noether Program (Grant No. RE 3977/1), and Grants No. FOR1700 (Project E5), No. SPP2244 (Project No. 443366970), and from the European Research Council, Grant (a)

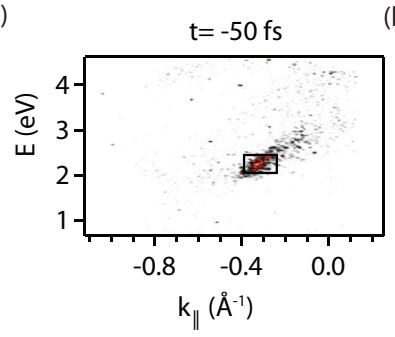

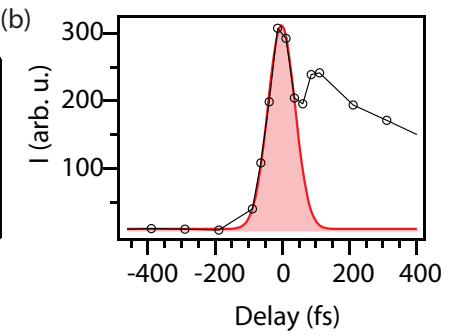

FIG. 5. (a) ARPES intensity as a function of energy and parallel momentum showing the conduction states along the $\bar{\Gamma}-\bar{\Sigma}$ direction at a time delay of $-50 \mathrm{fs}$. The pump-probe temporal crosscorrelation is determined by integrating the signal in the rectangular box. (b) Temporal trace showing the integrated intensity in the box of panel (a) as a function of time. Red curve, Gaussian fit to the rising edge, where the FWHM is 95 fs. 
No. ERC-2015-CoG-682843. M.P. acknowledges financial support from the Swiss National Science Foundation (SNSF) through Grant No. CRSK-2_196756. C.W.N. and C.M. acknowledge financial support by Swiss National Science Foundation (SNSF) Grant No. P00P2_170597. A.R. and H.H. acknowledge financial support from the European Research Council (Grant No. ERC-2015-AdG-694097) and the Cluster of Excellence "CUI: Advanced Imaging of Matter" of the Deutsche Forschungsgemeinschaft (Grant EXC 2056, Project No. 390715994).

\section{APPENDIX: DETERMINATION OF TEMPORAL PUMP-PROBE CROSS-CORRELATION}

The temporal time zero and pump-probe cross-correlation of $95 \mathrm{fs}$ were measured by fitting the rising edge of the first observable signal in the excited-state band structure, as illustrated in Fig. 5. The second maximum observed after $100 \mathrm{fs}$ is a result of electron population scattered from other states during the energy relaxation process.
[1] C. L. Smallwood, R. A. Kaindl, and A. Lanzara, Ultrafast angle-resolved photoemission spectroscopy of quantum materials, Europhys. Lett. 115, 27001 (2016).

[2] S. Dong, M. Puppin, T. Pincelli, S. Beaulieu, D. Christiansen, H. Hübener, C. W. Nicholson, R. P. Xian, M. Dendzik, Y. Deng, Y. W. Windsor, M. Selig, E. Malic, A. Rubio, A. Knorr, M. Wolf, L. Rettig, and R. Ernstorfer, Direct measurement of key exciton properties: Energy, dynamics, and spatial distribution of the wave function, Natural Sciences 1, e10010 (2021).

[3] U. De Giovannini, H. Hübener, S. A. Sato, and A. Rubio, Direct Measurement of Electron-Phonon Coupling with TimeResolved ARPES, Phys. Rev. Lett. 125, 136401 (2020).

[4] M. X. Na, A. K. Mills, F. Boschini, M. Michiardi, B. Nosarzewski, R. P. Day, E. Razzoli, A. Sheyerman, M. Schneider, G. Levy, S. Zhdanovich, T. P. Devereaux, A. F. Kemper, D. J. Jones, and A. Damascelli, Direct determination of mode-projected electron-phonon coupling in the time domain, Science 366, 1231 (2019).

[5] C. Ahn, A. Cavalleri, A. Georges, S. Ismail-Beigi, A. J. Millis, and J.-M. Triscone, Designing and controlling the properties of transition metal oxide quantum materials, Nat. Mater. 20, 1462 (2021)

[6] J. Güdde and U. Höfer, Femtosecond time-resolved studies of image-potential states at surfaces and interfaces of rare-gas adlayers, Prog. Surf. Sci. 80, 49 (2005).

[7] M. Weinelt, Time-resolved two-photon photoemission from metal surfaces, J. Phys.: Condens. Matter 14, R1099 (2002).

[8] M. Weinelt, M. Kutschera, T. Fauster, and M. Rohlfing, Dynamics of Exciton Formation at the $\operatorname{Si}(100) c(4 \times 2)$ Surface, Phys. Rev. Lett. 92, 126801 (2004).

[9] X. Y. Zhu, Photoemission from excitons in organic semiconductors, J. Electron Spectrosc. Relat. Phenom. 204, 75 (2015).

[10] J. A. Sobota, S.-L. Yang, A. F. Kemper, J. J. Lee, F. T. Schmitt, W. Li, R. G. Moore, J. G. Analytis, I. R. Fisher, P. S. Kirchmann, T. P. Devereaux, and Z.-X. Shen, Direct Optical Coupling to an Unoccupied Dirac Surface State in the Topological Insulator $\mathrm{Bi}_{2}$ $\mathrm{Se}_{3}$, Phys. Rev. Lett. 111, 136802 (2013).

[11] Y. H. Wang, H. Steinberg, P. Jarillo-Herrero, and N. Gedik, Observation of Floquet-Bloch states on the surface of a topological insulator, Science 342, 453 (2013).

[12] A. Rettenberger, P. Leiderer, M. Probst, and R. Haight, Ultrafast electron transport in layered semiconductors studied with femtosecond-laser photoemission, Phys. Rev. B 56, 12092 (1997).

[13] P. Hein, A. Stange, K. Hanff, L. X. Yang, G. Rohde, K. Rossnagel, and M. Bauer, Momentum-resolved hot electron dynamics at the $2 h-\mathrm{MoS}_{2}$ surface, Phys. Rev. B 94, 205406 (2016).

[14] R. Wallauer, J. Reimann, N. Armbrust, J. Güdde, and U. Höfer, Intervalley scattering in MoS2 imaged by two-photon photoemission with a high-harmonic probe, Appl. Phys. Lett. 109, 162102 (2016).

[15] S. C. J. Meskers, P. A. van Hal, A. J. H. Spiering, J. C. Hummelen, A. F. G. van der Meer, and R. A. J. Janssen, Timeresolved infrared-absorption study of photoinduced charge transfer in a polythiophene-methanofullerene composite film, Phys. Rev. B 61, 9917 (2000); R. Bertoni, C. W. Nicholson, L. Waldecker, H. Hubener, C. Monney, U. De Giovannini, M. Puppin, M. Hoesch, E. Springate, R. T. Chapman et al., Generation and Evolution of Spin-, Valley- and Layer-Polarized Excited Carriers in Inversion-Symmetric WSe2, Phys. Rev. Lett. 117, 277201 (2016).

[16] J. Madéo, M. K. L. Man, C. Sahoo, M. Campbell, V. Pareek, E. L. Wong, A. Al-Mahboob, N. S. Chan, A. Karmakar, B. M. K. Mariserla, X. Li, T. F. Heinz, T. Cao, and K. M. Dani, Directly visualizing the momentum-forbidden dark excitons and their dynamics in atomically thin semiconductors, Science 370, 1199 (2020).

[17] M. K. L. Man, J. Madéo, C. Sahoo, K. Xie, M. Campbell, V. Pareek, A. Karmakar, E. L. Wong, A. Al-Mahboob, N. S. Chan, D. R. Bacon, X. Zhu, M. M. M. Abdelrasoul, X. Li, T. F. Heinz, F. H. d. Jornada, T. Cao, and K. M. Dani, Experimental measurement of the intrinsic excitonic wave function, Sci. Adv. 7, eabg0192 (2021).

[18] S. Ulstrup, A. G. Čabo, J. A. Miwa, J. M. Riley, S. S. Grønborg, J. C. Johannsen, C. Cacho, O. Alexander, R. T. Chapman, E. Springate, M. Bianchi, M. Dendzik, J. V. Lauritsen, P. D. C. King, and P. Hofmann, Ultrafast band structure control of a twodimensional heterostructure, ACS Nano 10, 6315 (2016).

[19] S. Roth, A. Crepaldi, M. Puppin, G. Gatti, D. Bugini, I. Grimaldi, T. R. Barrilot, C. A. Arrell, F. Frassetto, L. Poletto, M. Chergui, A. Marini, and M. Grioni, Photocarrier-induced bandgap renormalization and ultrafast charge dynamics in black phosphorus, 2D Mater. 6, 031001 (2019).

[20] F. Liu, M. E. Ziffer, K. R. Hansen, J. Wang, and X. Zhu, Direct Determination of Band-Gap Renormalization in the Photoexcited Monolayer MoS2, Phys. Rev. Lett. 122, 246803 (2019).

[21] W. Lee, Y. Lin, L.-S. Lu, W.-C. Chueh, M. Liu, X. Li, W.-H. Chang, R. A. Kaindl, and C.-K. Shih, Time-resolved ARPES determination of a quasi-particle band gap and hot electron dynamics in monolayer MoS2, Nano Lett. 21, 7363 (2021). 
[22] J. H. Buss, H. Wang, Y. Xu, J. Maklar, F. Joucken, L. Zeng, S. Stoll, C. Jozwiak, J. Pepper, Y.-D. Chuang, J. D. Denlinger, Z. Hussain, A. Lanzara, and R. A. Kaindl, A setup for extremeultraviolet ultrafast angle-resolved photoelectron spectroscopy at 50-kHz repetition rate, Rev. Sci. Instrum. 90, 023105 (2019).

[23] M. Puppin, Y. Deng, C. W. Nicholson, J. Feldl, N. B. M. Schröter, H. Vita, P. S. Kirchmann, C. Monney, L. Rettig, M. Wolf, and R. Ernstorfer, Time- and angle-resolved photoemission spectroscopy of solids in the extreme ultraviolet at $500 \mathrm{kHz}$ repetition rate, Rev. Sci. Instrum. 90, 023104 (2019).

[24] E. J. Sie, T. Rohwer, C. Lee, and N. Gedik, Time-resolved XUV ARPES with tunable 24-33 eV laser pulses at $30 \mathrm{meV}$ resolution, Nat. Commun. 10, 3535 (2019).

[25] R. Cucini, T. Pincelli, G. Panaccione, D. Kopic, F. Frassetto, P. Miotti, G. M. Pierantozzi, S. Peli, A. Fondacaro, A. De Luisa, A. De Vita, P. Carrara, D. Krizmancic, D. T. Payne, F. Salvador, A. Sterzi, L. Poletto, F. Parmigiani, G. Rossi, and F. Cilento, Coherent narrowband light source for ultrafast photoelectron spectroscopy in the $17-31 \mathrm{eV}$ photon energy range, Struct. Dyn. 7, 014303 (2020).

[26] Y. Liu, J. E. Beetar, M. M. Hosen, G. Dhakal, C. Sims, F. Kabir, M. B. Etienne, K. Dimitri, S. Regmi, Y. Liu, A. K. Pathak, D. Kaczorowski, M. Neupane, and M. Chini, Extreme ultraviolet time- and angle-resolved photoemission setup with $21.5 \mathrm{meV}$ resolution using high-order harmonic generation from a turnkey Yb:KGW amplifier, Rev. Sci. Instrum. 91, 013102 (2020).

[27] A. Ciarrocchi, D. Unuchek, A. Avsar, K. Watanabe, T. Taniguchi, and A. Kis, Polarization switching and electrical control of interlayer excitons in two-dimensional van der Waals heterostructures, Nat. Photonics 13, 131 (2019).

[28] D. Unuchek, A. Ciarrocchi, A. Avsar, Z. Sun, K. Watanabe, T. Taniguchi, and A. Kis, Valley-polarized exciton currents in a van der Waals heterostructure, Nat. Nanotechnol. 14, 1104 (2019)

[29] Q. H. Wang, K. Kalantar-Zadeh, A. Kis, J. N. Coleman, and M. S. Strano, Electronics and optoelectronics of two-dimensional transition metal dichalcogenides, Nat. Nanotechnol. 7, 699 (2012).

[30] D. Kozawa, R. Kumar, A. Carvalho, K. Kumar Amara, W. Zhao, S. Wang, M. Toh, R. M. Ribeiro, A. H. Castro Neto, K. Matsuda, and G. Eda, Photocarrier relaxation pathway in twodimensional semiconducting transition metal dichalcogenides, Nat. Commun. 5, 4543 (2014).

[31] D. Y. Qiu, F. H. da Jornada, and S. G. Louie, Optical Spectrum of MoS2: Many-Body Effects and Diversity of Exciton States, Phys. Rev. Lett. 111, 216805 (2013).

[32] A. Damascelli, Angle-resolved photoemission studies of the cuprate superconductors, Rev. Mod. Phys. 75, 473 (2003).

[33] S. Moser, An experimentalist's guide to the matrix element in angle resolved photoemission, J. Electron Spectrosc. Relat. Phenom. 214, 29 (2017).

[34] S. Beaulieu, J. Schusser, S. Dong, M. Schüler, T. Pincelli, M. Dendzik, J. Maklar, A. Neef, H. Ebert, K. Hricovini, M. Wolf, J. Braun, L. Rettig, J. Minár, and R. Ernstorfer, Revealing Hidden Orbital Pseudospin Texture with Time-Reversal Dichroism in Photoelectron Angular Distributions, Phys. Rev. Lett. 125, 216404 (2020).

[35] S. Hüfner, Photoelectron Spectroscopy: Principles and Applications (Springer, Berlin, Heidelberg, 2003).
[36] M. Kotsugi, W. Kuch, F. Offi, L. I. Chelaru, and J. Kirschner, Microspectroscopic two-dimensional Fermi surface mapping using a photoelectron emission microscope, Rev. Sci. Instrum. 74, 2754 (2003).

[37] J. Maklar, S. Dong, S. Beaulieu, T. Pincelli, M. Dendzik, Y. W. Windsor, R. P. Xian, M. Wolf, R. Ernstorfer, and L. Rettig, A quantitative comparison of time-of-flight momentum microscopes and hemispherical analyzers for time- and angleresolved photoemission spectroscopy experiments, Rev. Sci. Instrum. 91, 123112 (2020).

[38] F. Boschini, D. Bugini, M. Zonno, M. Michiardi, R. P. Day, E. Razzoli, B. Zwartsenberg, M. Schneider, E. H. d. S. Neto, S. d. Conte, S. K. Kushwaha, R. J. Cava, S. Zhdanovich, A. K. Mills, G. Levy, E. Carpene, C. Dallera, C. Giannetti, D. J. Jones, G. Cerullo et al., Role of matrix elements in the time-resolved photoemission signal, New J. Phys. 22, 023031 (2020).

[39] S. W. Koch, M. Kira, G. Khitrova, and H. M. Gibbs, Semiconductor excitons in new light, Nat. Mater. 5, 523 (2006).

[40] S. Hellmann, K. Rossnagel, M. Marczynski-Bühlow, and L. Kipp, Vacuum space-charge effects in solid-state photoemission, Phys. Rev. B 79, 035402 (2009).

[41] M. Puppin, Y. Deng, O. Prochnow, J. Ahrens, T. Binhammer, U. Morgner, M. Krenz, M. Wolf, and R. Ernstorfer, $500 \mathrm{kHz}$ OPCPA delivering tunable sub-20 fs pulses with $15 \mathrm{~W}$ average power based on an all-ytterbium laser, Opt. Express 23, 1491 (2015).

[42] S. Sharma, C. Ambrosch-Draxl, M. A. Khan, P. Blaha, and S. Auluck, Optical properties and band structure of $2 h-\mathrm{WSe}_{2}$, Phys. Rev. B 60, 8610 (1999).

[43] J. P. Perdew, K. Burke, and M. Ernzerhof, Generalized Gradient Approximation Made Simple, Phys. Rev. Lett. 77, 3865 (1996).

[44] P. Giannozzi, S. Baroni, N. Bonini, M. Calandra, R. Car, C. Cavazzoni, D. Ceresoli, G. L. Chiarotti, M. Cococcioni, I. Dabo, A. D. Corso, S. de Gironcoli, S. Fabris, G. Fratesi, R. Gebauer, U. Gerstmann, C. Gougoussis, A. Kokalj, M. Lazzeri, L. Martin-Samos et al., QUANTUM ESPRESSO: A modular and open-source software project for quantum simulations of materials, J. Phys.: Condens. Matter 21, 395502 (2009).

[45] L. Hedin, New method for calculating the one-particle green's function with application to the electron-gas problem, Phys. Rev. 139, A796 (1965).

[46] F. Aryasetiawan and O. Gunnarsson, The GW method, Rep. Prog. Phys. 61, 237 (1998).

[47] J. Deslippe, G. Samsonidze, D. A. Strubbe, M. Jain, M. L. Cohen, and S. G. Louie, BerkeleyGW: A massively parallel computer package for the calculation of the quasiparticle and optical properties of materials and nanostructures, Comput. Phys. Commun. 183, 1269 (2012).

[48] J. M. Riley, F. Mazzola, M. Dendzik, M. Michiardi, T. Takayama, L. Bawden, C. Granerod, M. Leandersson, T. Balasubramanian, M. Hoesch, T. K. Kim, H. Takagi, W. Meevasana, P. Hofmann, M. S. Bahramy, J. W. Wells, and P. D. C. King, Direct observation of spin-polarized bulk bands in an inversion-symmetric semiconductor, Nat. Phys. 10, 835 (2014).

[49] T. Finteis, M. Hengsberger, T. Straub, K. Fauth, R. Claessen, P. Auer, P. Steiner, S. Hüfner, P. Blaha, M. Vögt, M. Lux-Steiner, and E. Bucher, Occupied and unoccupied electronic band structure of $\mathrm{WSe}_{2}$, Phys. Rev. B 55, 10400 (1997). 
[50] J. M. Riley, W. Meevasana, L. Bawden, M. Asakawa, T. Takayama, T. Eknapakul, T. K. Kim, M. Hoesch, S.-K. Mo, H. Takagi, T. Sasagawa, M. S. Bahramy, and P. D. C. King, Negative electronic compressibility and tunable spin splitting in $\mathrm{WSe}_{2}$, Nat. Nanotechnol. 10, 1043 (2015).

[51] I. Tanabe, T. Komesu, D. Le, T. B. Rawal, E. F. Schwier, M. Zheng, Y. Kojima, H. Iwasawa, K. Shimada, T. S. Rahman, and P. A. Dowben, The symmetry-resolved electronic structure of 2H-WSe 2 (0001), J. Phys.: Condens. Matter 28, 345503 (2016).

[52] S. Hüfner, Very High Resolution Photoelectron Spectroscopy (Springer, Berlin, 2007).

[53] A. D. Katnani and G. Margaritondo, Microscopic study of semiconductor heterojunctions: Photoemission measurement of the valance-band discontinuity and of the potential barriers, Phys. Rev. B 28, 1944 (1983).

[54] J. C. Fuggle, Unoccupied Electronic States: Fundamentals for $X A N E S, E E L S, I P S$ and BIS (Springer, Berlin, Heidelberg, 1992).

[55] F. J. Himpsel, Inverse photoemission from semiconductors, Surf. Sci. Rep. 12, 3 (1990).

[56] N. D. Lang, Spectroscopy of single atoms in the scanning tunneling microscope, Phys. Rev. B 34, 5947 (1986).

[57] V. N. Strocov, Very low-energy electron diffraction as a method of band structure investigations: Applications in photoelectron spectroscopy, Phys. Solid State 42, 1973 (2000).

[58] L. Hedin and S. Lundqvist, Effects of Electron-Electron and Electron-Phonon Interactions on the One-Electron States of Solids (Academic Press, New York, 1970), pp. 1-181.

[59] B. S. Kim, J.-W. Rhim, B. Kim, C. Kim, and S. R. Park, Determination of the band parameters of bulk $2 \mathrm{H}-\mathrm{MX}_{2}(\mathrm{M}=\mathrm{Mo}$, $\mathrm{W} ; \mathrm{X}=\mathrm{S}, \mathrm{Se}$ ) by angle-resolved photoemission spectroscopy, Sci. Rep. 6, 36389 (2016).

[60] C. Monney, M. Herzog, A. Pulkkinen, Y. Huang, J. Pelliciari, P. Olalde-Velasco, N. Katayama, M. Nohara, H. Takagi, T. Schmitt, and T. Mizokawa, Mapping the unoccupied state dispersions in $\mathrm{Ta}_{2} \mathrm{NiSe}_{5}$ with resonant inelastic x-ray scattering, Phys. Rev. B 102, 085148 (2020).

[61] C. Monney, K. J. Zhou, H. Cercellier, Z. Vydrova, M. G. Garnier, G. Monney, V. N. Strocov, H. Berger, H. Beck, T. Schmitt, and P. Aebi, Mapping of Electron-Hole Excitations in the Charge-Density-Wave System 1T- $\mathrm{TiSe}_{2}$ Using Resonant Inelastic X-Ray Scattering, Phys. Rev. Lett. 109, 047401 (2012).

[62] M. Traving, M. Boehme, L. Kipp, M. Skibowski, F. Starrost, E. E. Krasovskii, A. Perlov, and W. Schattke, Electronic structure of $\mathrm{WSe}_{2}$ : A combined photoemission and inverse photoemission study, Phys. Rev. B 55, 10392 (1997).

[63] A. R. Beal and W. Y. Liang, Excitons in $2 \mathrm{H}-\mathrm{WSe}_{2}$ and $3 \mathrm{R}-\mathrm{WS}_{2}$, J. Phys. C: Solid State Phys. 9, 2459 (1976).

[64] H. Zeng, G.-B. Liu, J. Dai, Y. Yan, B. Zhu, R. He, L. Xie, S. Xu, X. Chen, W. Yao, and X. Cui, Optical signature of symmetry variations and spin-valley coupling in atomically thin tungsten dichalcogenides, Sci. Rep. 3, 1608 (2013).

[65] A. Arora, M. Koperski, K. Nogajewski, J. Marcus, C. Faugeras, and M. Potemski, Excitonic resonances in thin films of $\mathrm{WSe}_{2}$ : From monolayer to bulk material, Nanoscale 7, 10421 (2015).

[66] R. Schuster, Y. Wan, M. Knupfer, and B. Büchner, Nongeneric dispersion of excitons in the bulk of $\mathrm{WSe}_{2}$, Phys. Rev. B 94, 085201 (2016).
[67] A. Jain, S. P. Ong, G. Hautier, W. Chen, W. D. Richards, S. Dacek, S. Cholia, D. Gunter, D. Skinner, G. Ceder, and K. A. Persson, The Materials Project: A materials genome approach to accelerating materials innovation, APL Mater. 1, 011002 (2013).

[68] R. Roldán, J. A. Silva-Guillén, M. P. López-Sancho, F. Guinea, E. Cappelluti, and P. Ordejón, Electronic properties of singlelayer and multilayer transition metal dichalcogenides $M X_{2}$ ( $M=\mathrm{Mo}, \mathrm{W}$ and $X=\mathrm{S}, \mathrm{Se})$, Ann. Phys. (Berlin) 526, 347 (2014).

[69] A. Kumar and P. K. Ahluwalia, Electronic structure of transition metal dichalcogenides monolayers $1 \mathrm{H}-\mathrm{MX}_{2}(\mathrm{M}=\mathrm{Mo}, \mathrm{W} ; \mathrm{X}=$ $\mathrm{S}, \mathrm{Se}, \mathrm{Te}$ ) from ab-initio theory: New direct band gap semiconductors, Eur. Phys. J. B 85, 186 (2012).

[70] W. Huang, X. Luo, C. K. Gan, S. Y. Quek, and G. Liang, Theoretical study of thermoelectric properties of few-layer $\mathrm{MoS}_{2}$ and $\mathrm{WSe}_{2}$, Phys. Chem. Chem. Phys. 16, 10866 (2014).

[71] S. Curtarolo, W. Setyawan, S. Wang, J. Xue, K. Yang, R. H. Taylor, L. J. Nelson, G. L. W. Hart, S. Sanvito, M. BuongiornoNardelli, N. Mingo, and O. Levy, AFLOWLIB.ORG: A distributed materials properties repository from high-throughput ab initio calculations, Comput. Mater. Sci. 58, 227 (2012).

[72] H. Jiang, Electronic band structures of molybdenum and tungsten dichalcogenides by the GW approach, J. Phys. Chem. C 116, 7664 (2012).

[73] J. He, K. Hummer and C. Franchini, Stacking effects on the electronic and optical properties of bilayer transition metal dichalcogenides $\mathrm{MoS}_{2}, \mathrm{MoSe}_{2}, \mathrm{WS}_{2}, \mathrm{WSe}{ }_{2}$, Phys. Rev. B 89, 075409 (2014).

[74] E. J. Baerends, O. V. Gritsenko, and R. v. Meer, The KohnSham gap, the fundamental gap and the optical gap: The physical meaning of occupied and virtual Kohn-Sham orbital energies, Phys. Chem. Chem. Phys. 15, 16408 (2013).

[75] F. Giustino, Materials Modelling Using Density Functional Theory: Properties and Predictions (Oxford University Press, Oxford, 2014).

[76] J. P. Perdew and M. Levy, Physical Content of the Exact Kohn-Sham Orbital Energies: Band Gaps and Derivative Discontinuities, Phys. Rev. Lett. 51, 1884 (1983).

[77] T. Straub, K. Fauth, T. Finteis, M. Hengsberger, R. Claessen, P. Steiner, S. Hufner, and P. Blaha, Valence-band maximum in the layered semiconductor $\mathrm{WSe}_{2}$ : Application of constantenergy contour mapping by photoemission, Phys. Rev. B 53, 016152(R) (1996).

[78] J. P. Perdew, Density functional theory and the band gap problem, Int. J. Quantum Chem. 28, 497 (2009).

[79] M. S. Hybertsen and S. G. Louie, Electron correlation in semiconductors and insulators: Band gaps and quasiparticle energies, Phys. Rev. B 34, 5390 (1986).

[80] M. Rohlfing and S. G. Louie, Electron-hole excitations and optical spectra from first principles, Phys. Rev. B 62, 4927 (2000).

[81] E. Perfetto, D. Sangalli, A. Marini, and G. Stefanucci, First-principles approach to excitons in time-resolved and angle-resolved photoemission spectra, Phys. Rev. B 94, 245303 (2016).

[82] A. Rustagi and A. F. Kemper, Photoemission signature of excitons, Phys. Rev. B 97, 235310 (2018).

[83] D. Christiansen, M. Selig, E. Malic, R. Ernstorfer, and A. Knorr, Theory of exciton dynamics in time-resolved ARPES: 
Intra- and intervalley scattering in two-dimensional semiconductors, Phys. Rev. B 100, 205401 (2019).

[84] J. Shah, Ultrafast Spectroscopy of Semiconductors and Semiconductor Nanostructures (Springer Verlag, Berlin, 2010).

[85] L. Waldecker, R. Bertoni, H. Hübener, T. Brumme, T. Vasileiadis, D. Zahn, A. Rubio, and R. Ernstorfer, MomentumResolved View of Electron-Phonon Coupling in Multilayer $\mathrm{WSe}_{2}$, Phys. Rev. Lett. 119, 036803 (2017).

[86] W. S. Yun, S. W. Han, S. C. Hong, I. G. Kim, and J. D. Lee, Thickness and strain effects on electronic structures of transition metal dichalcogenides: $2 \mathrm{H}-\mathrm{MX}_{2}$ semiconductors $(\mathrm{M}=$ M0, W; X = S, Se, Te), Phys. Rev. B 85, 033305 (2012).

[87] M. Puppin, S. Polishchuk, N. Colonna, A. Crepaldi, D. N. Dirin, O. Nazarenko, R. De Gennaro, G. Gatti, S. Roth, T. Barillot, L. Poletto, R. P. Xian, L. Rettig, M. Wolf, R. Ernstorfer, M. V. Kovalenko, N. Marzari, M. Grioni, and M. Chergui, Evidence of Large Polarons in Photoemission Band Mapping of the Perovskite Semiconductor $\mathrm{CsPbBr}_{3}$, Phys. Rev. Lett. 124, 206402 (2020).

[88] A. A. Mitioglu, P. Plochocka, ÃA. Granados del Aguila, P. C. M. Christianen, G. Deligeorgis, S. Anghel, L. Kulyuk, and D. K. Maude, Optical investigation of monolayer and bulk tungsten diselenide $\left(\mathrm{WSe}_{2}\right)$ in high magnetic fields, Nano Lett. 15, 4387 (2015).

[89] J. R. Schaibley, H. Yu, G. Clark, P. Rivera, J. S. Ross, K. L. Seyler, W. Yao, and X. Xu, Valleytronics in 2D materials, Nat. Rev. Mater. 1, 16055 (2016).

[90] F. A. Rasmussen and K. S. Thygesen, Computational 2D materials database: Electronic structure of transition-metal dichalcogenides and oxides, J. Phys. Chem. C 119, 13169 (2015).
[91] R. P. Xian, V. Stimper, M. Zacharias, S. Dong, M. Dendzik, S. Beaulieu, B. Schölkopf, M. Wolf, L. Rettig, C. Carbogno, S. Bauer, and R. Ernstorfer, A machine learning route between band mapping and band structure, arXiv:2005.10210 v1.

[92] J. Maklar, Y. W. Windsor, C. W. Nicholson, M. Puppin, P. Walmsley, V. Esposito, M. Porer, J. Rittmann, D. Leuenberger, M. Kubli, M. Savoini, E. Abreu, S. L. Johnson, P. Beaud, G. Ingold, U. Staub, I. R. Fisher, R. Ernstorfer, M. Wolf, and L. Rettig, Nonequilibrium charge-density-wave order beyond the thermal limit, Nat. Commun. 12, 2499 (2021).

[93] C. W. Nicholson, M. Puppin, A. Lücke, U. Gerstmann, M. Krenz, W. G. Schmidt, L. Rettig, R. Ernstorfer, and M. Wolf, Excited-state band mapping and momentum-resolved ultrafast population dynamics in $\operatorname{In} / \mathrm{Si}(111)$ nanowires investigated with XUV-based time- and angle-resolved photoemission spectroscopy, Phys. Rev. B 99, 155107 (2019).

[94] C. W. Nicholson, A. Lücke, W. G. Schmidt, M. Puppin, L. Rettig, R. Ernstorfer, and M. Wolf, Beyond the molecular movie: Dynamics of bands and bonds during a photoinduced phase transition, Science 362, 821 (2018).

[95] S.-L. Yang, J. A. Sobota, Y. He, Y. Wang, D. Leuenberger, H. Soifer, M. Hashimoto, D. H. Lu, H. Eisaki, B. Moritz, T. P. Devereaux, P. S. Kirchmann, and Z.-X. Shen, Revealing the Coulomb interaction strength in a cuprate superconductor, Phys. Rev. B 96, 245112 (2017).

[96] Y. Wang, M. Claassen, C. D. Pemmaraju, C. Jia, B. Moritz, and T. P. Devereaux, Theoretical understanding of photon spectroscopies in correlated materials in and out of equilibrium, Nat. Rev. Mater. 3, 312 (2018). 